\title{
Impact of thymoquinone supplementation on immobilisation stress-induced changes in reproductive characteristics of male mice
}

\author{
Suzanah Abdul Rahman*, Norul Athirah Mohamed Anwar, Shaik Sadak Basha and \\ Saheera Kamarzaman
}

Department of Biomedical Science, Faculty of Allied Health Sciences, International Islamic University Malaysia, MALAYSIA

*Corresponding author. E-mail: suzanahrahman@yahoo.com

Received: September 22, 2013; Revised received: October 16, 2013; Accepted: January 3, 2014

\begin{abstract}
The aim of the current study was to investigate changes in the reproductive parameters during stress and the impact of thymoquinone during the period. The effects of stress were measured through immobilisation stress on mice. Group I was administered normal saline daily via intraperitoneal injection while Groups II and III were subjected to 2 and 6 hours of immobilisation stress respectively. Groups IV and V were subjected to stress for 2 and 6 hours respectively followed by intraperitoneal injection of $10 \mathrm{mg} / \mathrm{kg}$ thymoquinone which was continued on alternate days. The level of significance was set at $p<0.05$ and statistical analysis showed significant difference in testicular weight of mice in groups II and III compared to the controls but no significant difference was obtained for sperm count between all groups. Sperm motility, however, was significantly different among the groups under stress for 2 and 6 hours and that of 6 hours with the treatment of thymoquinone when compared to the controls. The histology of the testes also indicated a few alterations in comparison to the controls in the germinal epithelium and spermatogenic pattern in groups III and V.
\end{abstract}

Keywords: Histopathology, Immobilisation Stress, Male mice, Sperm, Testis, Thymoquinone

\section{INTRODUCTION}

Stress in the world today is a lifetime tribulation for human beings. The term stress has now become a most widely used vocabulary to capture a variety of human experiences that are disturbing in some manner. Stress associated with workplace, family and other factors has been implicated as one of the factors to be involved in the severity and progression of a number of diseases (Ameen, 2009). Stress has become one of the major concerns among the human population. Stress related problems consistently exist in societies around the world and the impact on human health is seen to be rising globally. Stress precipitates many neuropsychiatric parameters, such as anxiety, depression and memory loss and cause antioxidant imbalance. Indeed, there have been hundreds of empirical reports of the association between stressful life events and health outcomes (Avison and Gotlib, 1994).

In addition, Eriksen and Ursin (2002) defined stress as a state of threatened homeostasis or disharmony and it is counteracted by a complex selective physiologic and behavioral responses. It is believed that stress has many impacts on the mechanism of human body which may alter the body's normal biological system. Recent experimental studies have shown that there is a relationship between stress and human semen quality
(Ameen, 2009) and stress may suppress sexual or reproductive function (Woo et al., 2011). According to Khandve et al. (2013) stress is an aversive stimulus which disturbs physiological homeostasis. Stress precipitates many neuropsychiatric parameters, such as anxiety, depression and memory loss and cause antioxidant imbalance. The situation is further supported by hundreds of empirical reports showing association between stressful life events and adverse health outcomes (Avison and Gotlib, 1994).

It is reported that Nigella sativa exhibits a significant level of anti-stress activity in albino rats and has shown analgesic activity in mice (Roshan et al., 2010). Thymoquinone (TQ), an active ingredient of Nigella sativa, has been reported to exhibit many pharmacological effects including as an immunomodulator (Salem, 2005) anticancer (Tavakoli et al., 2012), anti-diabetic (Bouchra et al., 2009), anti-oxidant and anti-inflammatory (Mohammad et al., 2009) agent. Studies have reported that TQ can protect several organs against oxidative damage but only a few data are available concerning its effect on stress. This research is done to further elucidate the potential benefits of TQ and to observe the effect of supplementation of TQ on immobilisation stressinduced changes in reproductive characteristics of the male mouse. 


\section{MATERIALS AND METHODS}

Experimental animals: The experiment was performed on adult male BALB/c (albino) mice weighing 25-30 gm. All of the animals were allowed to acclimatize in metal cages inside a well-ventilated room for 2 weeks prior to the experiment. They were housed in groups of six per each polycarbonate cage under standard laboratory conditions at a room temperature of $22 \pm 4^{0} \mathrm{C}$, humidity of $30-70 \%$ with 12 hours light/dark cycle. The animals were fed with standard diet and tap water was given ad libitum until treatment or time of sacrifice. The study was performed according to the guidelines for animal study and was approved by the Faculty of Allied Health Sciences Research Committee of the institution.

Experimental design: The animals were divided into five groups. Group I mice were controls injected with normal saline via intraperitoneal (i.p.) injection while animals in groups II and III were subjected to immobilisation stress for 2 and 6 hours respectively. Groups IV and V were also subjected to immobilisation stress for 2 and 6 hours respectively but animals in both groups later received i.p. injection of $10 \mathrm{mg} / \mathrm{kg}$ of TQ. Immobilisation stress for all groups involved were applied 5 days a week for a total period of 32 days. Supplementation of TQ for groups IV and $V$ was continued on alternate days for the length of the study. All the mice were sacrificed at day 32 via cervical dislocation to examine the testicular weight, sperm count, sperm motility and morphology of testes.

Stress was applied through immobilisation of the mice. Immobilisation stress acts as physical and psychological stress (Khandve et al., 2013). The mice were immobilised by placing them into a handmade cones which were made using an autoclave plastic bag with the tip cut to form a hole for ventilation. Once the mouse was placed into the cone, the sides of the bag were stapled neatly in order to immobilise the mice completely.

Epididymal sperm collection and sperm preparation: Mice were dissected and their epididymis removed and placed into $1 \mathrm{ml}$ sperm preparation tubes with medium. The tube caps were loosen and tubes were immediately placed into the $\mathrm{CO}_{2}$ incubator. After 30 minutes, the sperm preparation medium tubes containing the epididymis were removed from the incubator. A cloudy preparation indicated that sperm had been released from the epididymis into the medium. A pipette was used to remove up to $1 \mathrm{ml}$ of sperm preparation medium and mixed with the sperm from the top of medium which was known to contain the highest quantity of motile sperm.

Sperm count and motility: An approximate number of sperm was calculated using the haemocytometer. $10 \mu \mathrm{l}$ of sperm was taken from the preparation and were placed into the counting chamber of the haemocytometer and covered with a cover slip and viewed under a light microscope. Sperm count was taken by calculating the number of sperm in five randomly picked boxes from the counting chamber. Sperm motility was also calculated.

Histology: Testis was preserved in $10 \%$ of neutral buffered formalin immediately after their removal from the animal for 1 hour and tissues were dehydrated in an ascending grade of alcohol and later three changes of $100 \%$ alcohol for 1.5 hours each. Dehydration followed with immersion in equal parts of alcohol and toluene for 30 minutes and toluene alone overnight. The dehydrated tissues were then embedded in paraffin blocks using wax of the same grade. The paraffin blocks were mounted and sections were later cut using a rotary microtome at a $3 \mu \mathrm{m}$ thickness and mounted on labelled slides.

Following floatation process and adequate drying, the paraffin was first melted in an incubator at $60^{\circ} \mathrm{C}$ for 10 minutes and sections were allowed to cool before staining. Sections were later deparaffinized by immersion in two changes of xylene for 5 minutes followed by washing in $100 \%$ alcohol and descending solutions of alcohol each for 3 minutes. Staining commenced with immersion in Ehrlich's hematoxylin for 15 minutes followed by counter-staining in $1 \%$ aqueous eosin for 1 minute. Excess of both stains were removed by washing with tap water following the normal procedures and sections were dehydrated with ascending grades of alcohol ending with two washes with xylene. When the sections were cooled, they were mounted in DPX and dried overnight. The architecture of the sectioned tissues was later observed using a low-power objective under a microscope.

Statistical analysis: All the data are expressed using mean \pm standard error of mean (S.E.M). Statistical differences between the groups were analysed using one-way ANOVA followed by Tukey's Post Hoc test using the SPSS version 12.0. The differences were considered statistically significant at $p<0.05$.

\section{RESULTS AND DISCUSSION}

Testicular weight, sperm count and motility: Stress is believed to play a role in semen quality changes and damage to reproductive organs (Al-Zahrani et al., 2012). Fig.1 shows the comparisons of testicular weight of $\mathrm{Balb} / \mathrm{c}$ mice between controls and the experimental groups which consisted of groups of stress for 2 hours, 2 hours treated with TQ, 6 hours and 6 hours treated with TQ. There was a significant difference $(p<0.05)$ in the testicular weight of mice between the control group and that subjected to stress for 6hours.Based on these results, a reduction in testicular weight of controls when compared to the group under immobilisation stress for 6 hours seemed to contradict the study by Roshan et al. (2010), that reported mice exposed to immobilisation stress have 


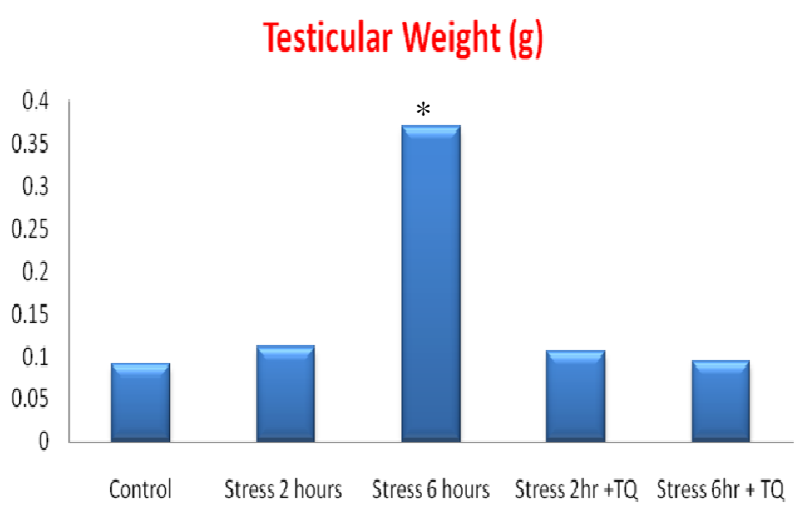

Fig. 1. Testicular weight ( $g$ ) of Balb/c mice in the control and experimental groups. The results are presented in mean of replicates. $* p<0.05$ significantly different as compared to controls.

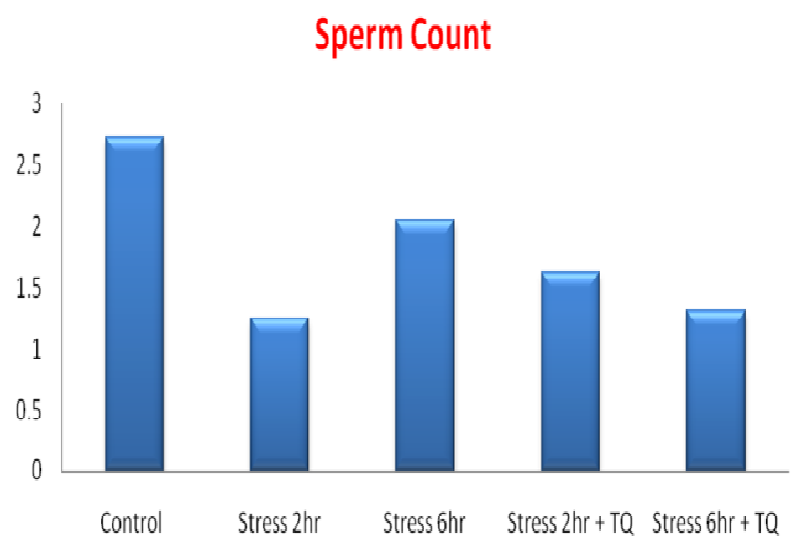

Fig. 2. Sperm count (million/ml) of Balb/c mice in the control and experimental groups. The results are presented in mean of replicates.

reduced testicular weights compared to the control group. Tissue adaptation to physical stress include hypertrophy indicating increased stress tolerance (Mueller and Maluf, 2002) and the testicular weight increase for the longer period of stress application may well be characteristic of tissue response but not necessarily due to an increase of the number of constituent cells.

There was no significant reduction in sperm count between any of the groups when compared to the controls as shown in fig. 2 . Results also indicated that there was no positive effect from TQ which was believed to increase the sperm count as mentioned by Mohammad et al. (2009). Immobilisation stress for 2 hours/day would not be adequate to produce intense stress on the mice (Bakhtiar et al., 2013) and mice could have adapted well during the immobilised state and thus may not really have an effect on sperm quality. Eriksen and Ursin (2002) stated that the body re-establishes the altered homeostasis by various behavioral adaptive responses. Thus, this could be a possible explanation for such observation in the sperm count parameter of the group under stress for 2 hours which seemed to indicate the most reduced number when compared to controls.
Sperm Motility (\%)

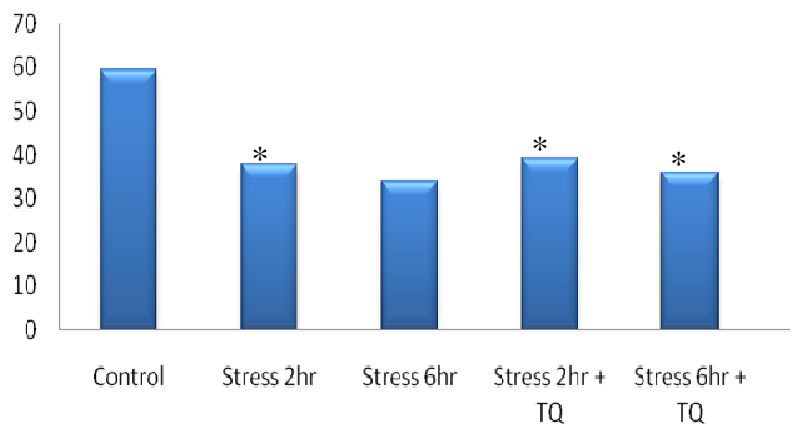

Fig. 3. The percentage of sperm motility (\%) of Balb/c mice in the control and experimental groups. The results are presented in mean of replicates. $* p<0.05$ significantly different as compared to control.

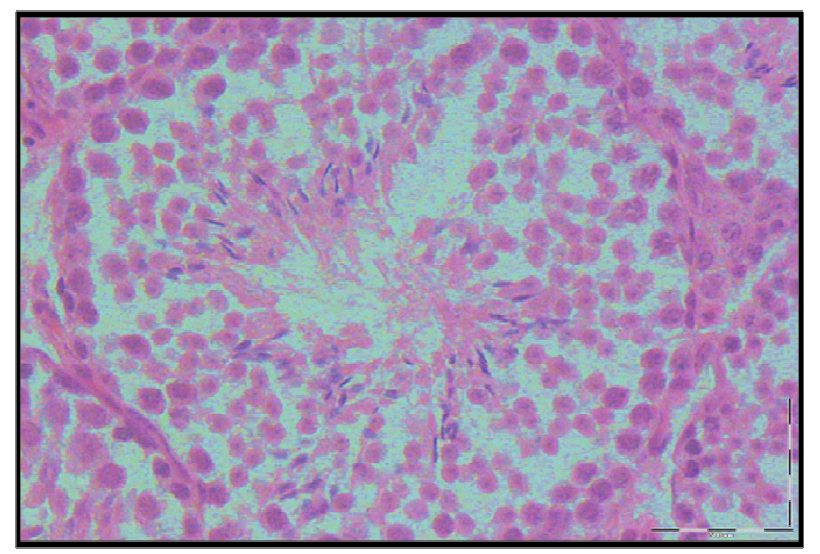

Fig. 4. Germinal epithelium of the seminiferous tubule in the testis of a Balb/c mouse in the control group at 32 days. $(H \&$ $E ; X 40)$.

The results for sperm motility between control and the experimental groups are shown in fig. 3. There were significant reductions $(p<0.05)$ in sperm motility of the groups of stress for 2 hours, 6 hours and 6 hours treated with TQ when compared to the controls. These findings were in accordance with Mesembe et al. (2009), who reported that long term exposure to stress affects sperm motility. Similarly Ren et al. (2010) also reported that restrain stress rapidly suppressed the sperm motility. Mohammad et al. (2009) showed that supplementation of TQ in mice significantly increased sperm motility and Al-Zahrani et al. (2012) has also shown that TQ increase the sperm quality of mice. However, results for sperm motility for the group applied with stress for 6 hours and that supplemented with TQ after 6 hours of immobilisation did not differ much in value indicating TQ may not have had a significant effect on the motility functions of the sperm produced.

Histology of testis: The effect of stress and treatment with TQ were further demonstrated by the histopathological changes of the testis. Based on Fig. 4 , testis of the control mouse showed regularly arranged germinal epithelium of seminiferous tubules and normal spermatogenesis which consist of all the 


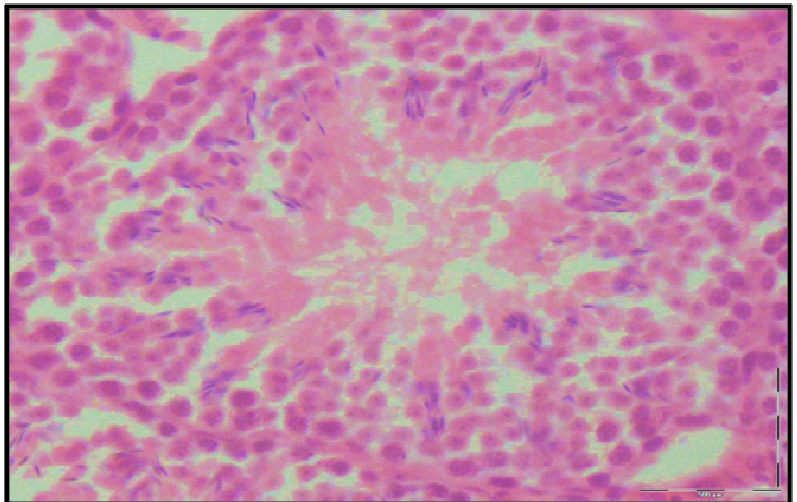

Fig. 5. Germinal epithelium of the seminiferous tubulein the testis of a Balb/c mouse applied immobilisation stress for 2 hours weekly at 32days. (H and E; X40).

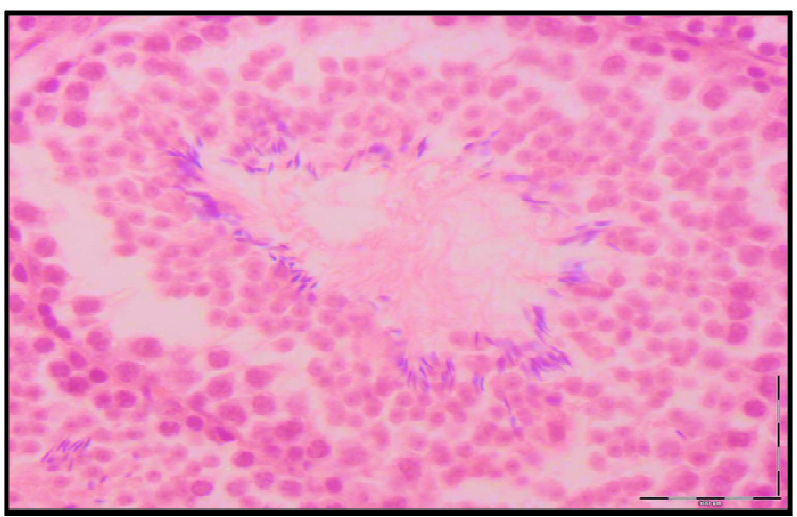

Fig. 6. Germinal epithelium of the seminiferous tubule in the testis of a Balb/c mouse applied immobilisation stress for 2 hours weekly and treatment with $10 \mathrm{mg} / \mathrm{kg}$ thymoquinone at 32 days. (H and $E ; X 40)$.

cells of the spermatogenic epithelium. Similar observations have been reported by Khandve et al. (2013) who investigated the effects of immobilisation stress on the spermatogenesis process of adult Swiss Albino mice. Based on figs. 5 and 6 and coinciding with the sperm count data, all the seminiferous tubules in the groups of mice influenced by immobilisation stress for 2 hours and those treated with TQ showed presence of spermatozoa but they were reduced in number as compared to the controls. Results seemed to suggest that mice under stress for 2 hours daily have sperm production that may have been affected and treatment with TQ may not have had an ameliorating effect on the number of sperm. Meanwhile, all the seminiferous tubules under the impact of stress for 6 hours (Fig. 7) showed slight reduction in size and the germinal epithelium of the tubules seemed disorganized. The lumen of the tubules was seen to be vacuolated and reductions in the number of spermatogonia, spermatocytes, spermatids and spermatozoa can be clearly seen in the seminiferous tubules. Tavakoli et al.(2012) also observed deformed seminiferous tubules and decreased number of spermatids, spermatocytes and spermatozoa in the restrained mice compared to controls. This suggests

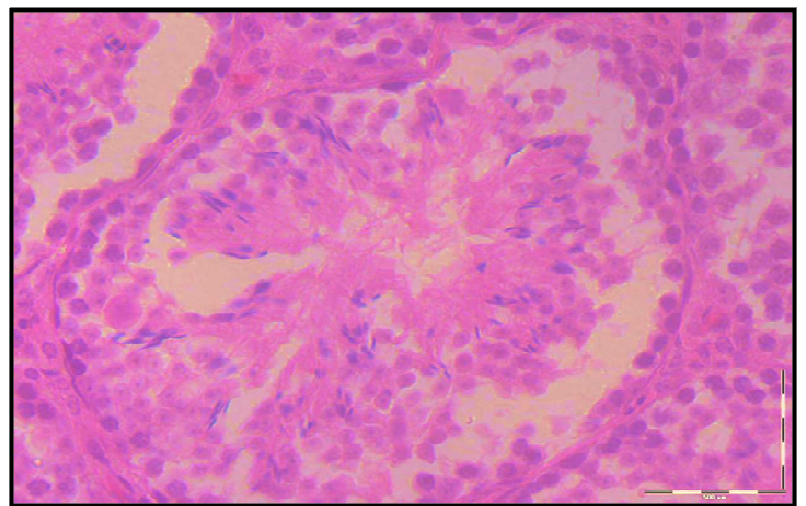

Fig.7. Germinal epithelium of the seminiferous tubule in the testis of a Balb/c mouse under immobilisation stress for 6 hours weekly at 32 days. (H and E; X40).

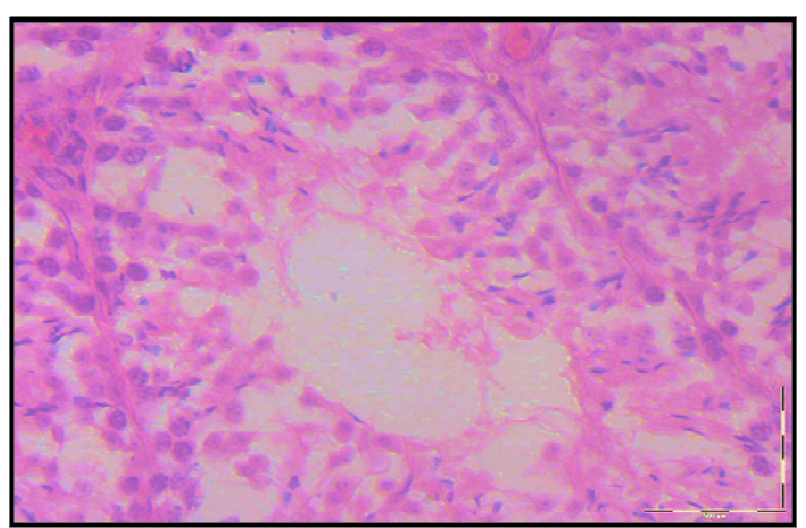

Fig. 8. Germinal epithelium of the seminiferous tubule in the testis of a Balb/c mouse under immobilisation stress for 6 hours weekly and treatment with $10 \mathrm{mg} / \mathrm{kg}$ thymoquinone at 32 days. ( $H$ and $E ; X 40)$.

that there were disturbances in the interior surroundings of tubules and suppression of spermatogenesis in the stressed groups (Khandve et al., 2013). The reason for suppressed spermatogenesis may be due to the immobilisation stress that could have induced depression of the hypothalamus-pituitary -testis axis which led to the activation of the hypothalamus pituitary-adrenocorticol axis resulting in reduced plasma luteinizing hormone and testosterone levels as reported by Khandve et al. (2013). Testosterone levels in the body play an important role in maintaining normal spermatogenesis and lower testosterone level can result in reduced sperm count (Zhang et al.,2010).

The seminiferous tubules in the group applied with stress for 6 hours and later treated with TQ (Fig. 8 ) showed vacuolated lumen and disorganized tubules which seemed to be similar to the effects of stress for 6 hours (Fig. 7). A possible explanation for such changes in the seminiferous tubules might be due to the fall in the plasma testosterone level which may have altered the metabolic activity of all the cells in the tubules. However, treatment with TQ did not seem to produce any recovery in the tubules. Contradictory to this, Al-Zahrani et al. (2012) observed that seminiferous 
tubules of Swiss Albino mice under heat stress when supplemented with TQ at $5 \mathrm{mg} / \mathrm{kg}$ a day for 75 days showed recovery in the structure of germinal epithelium and improved arrangement of cells in the seminiferous tubules. The said findings suggest that a longer duration of treatment with TQ may have a role in the recovery of spermatogenic cells and the epithelium.

\section{Conclusion}

In conclusion to our observations, immobilisation stress for 32 days had been shown to cause disturbance in the process of spermatogenesis and the germinal epithelium of the Balb/c mouse. Disorganised germinal epithelium of seminiferous tubule and the reduction in the height of germinal epithelium such as that seen in the histological observations including the decreased number of sperm count and reduced sperm motility may suggest suppression of hypothalamic-pituitary testicular system when mice were exposed to immobilisation stress. Knowledge on the longer term effects of thymoquinone supplementation and its role in the protection of reproductive tissues against stress could be worth further investigation.

\section{ACKNOWLEDGEMENT}

This paper was partially funded by the International Islamic University Malaysia Endowment fund and authors would like to acknowledge the statistical assistance from Assistant Professor Dr. Nor Azlina Ab. Rahman from the Department of Biomedical Science, Faculty of Allied Health Sciences and the technical help by Madam Sriviowarti Noerdin from the Faculty of Pharmacy and Sr. Azantee Yazmie Abdul Wahab from the Fertility Centre of International Islamic University Malaysia.

\section{REFERENCES}

Al-Zahrani, S., Mohany, M., Kandeal, S. and Badr, G. (2012). Thymoquinone and Vitamin E supplementation improve the reproductive characteristics of heat stressed male mice. Journal of Medicinal Plants Research, 6(3): 493-499.

Ameen, E.M. (2009). Effect of stress on semen quality in a population of infertile Human. Kufa Medical Journal, 12(1):167-174

Avison, W.R. and Gotlib, I.H. (1994). Stress and mental health contemporary issues and prospects for the future. New York; Plenum Press.

Bakhtiar, M. T., Mat Alewi, Nur Aizura, Darnis, Deny Susanti, Zamli, Zaitunnatakhin, Ramli, Norazsida, Saad, and Nurulwahida. (2013). A new sesquiterpene from Knemapatentinervia, Chemistry of Natural Compounds, 48 (6): 985-987.

Bouchra, M., Robert, D., Moulay, F., Bruno, E.,Lahcen, M. and Ali, B. (2009). Nigella sativa inhibits intestinal glucose absorption and improves glucose tolerance in rats. J. Ethnopharmacology, 121(3): 419-424.

Eriksen, H.R and Ursin, H. (2002). Sensitization and subjective health complaints. Scand. J. of Psychol., 43 (2):189-96.

Khandve, B., Gujar, V., Bokariya, P.,Tarnekar, A. and Shende, M. (2013). Deranged spermatogenesis of adult Swiss Albino Mice as Effect of Immobilisation Stress histological study. Journal of Pharmacy, 3 (2):7-10.

Mesembe, O.E., Igiri, A.O., Fischer, V.A.,Bisong, S.A. and Ekam, V.S. (2009). Sperm quality and fertility following Prolonged Immobilisation Stress in Wistar Rats. Mary Slessor. Journal of Medicine, 9 (1):http:// dx.doi.org/10.4314/msjm.v9i1.46649.

Mohammad, M.A., Mohamad, M.M. and Dradka, H. (2009). Effects of black seeds (Nigella sativa) on spermatogenesis and fertility of male Albino rats. Research Journal of Medicine and Medical Sciences, 4 (2): 386-390.

Mueller, M.J. and Maluf, K. S. (2002). Tissue adaptation to physical stress: A proposed "Physical Stress Theory" to guide physical therapist practice. Education and Research, 82 (4):383-403.

Ren, L., Li, X., Weng, Q.,Trisomboon, H., Pan, L., Watanabe, G. and Taya, K. (2010). Effects of acute restrain stress on sperm motility and secretion of pituitary, adrenocorticol and gonadal hormones in adult male rats. The Journal of Veterinary Medical Science, 72(11):1501-1506.

Roshan, S., Khan, A., Tazneem, B. and Ali, S. (2010). To study the effect of Nigella sativa on various biochemical parameters on stress induced in Albino rats. International Journal of Pharmacy and Pharmaceutical Science, 2 (4):185-189.

Salem, M.L. (2005). Immunomodulatory and therapeutic properties of the Nigella sativa L. seed. Epub. 5(1314):1749-79. PMID:16275613.

Tavakoli, P., Ahmadi, R., Mafi, M. (2012). Restraint stress is biomedically important in male reproductive failure. International Conference on Chemical. Biological and Medical Sciences Kuala Lumpur Malaysia ICCBMS.

Woo, C.C., Loo, S.Y., Gee, V., Yap, C.W., Sethi, G., Kumar, A.P. and Tan, K.H. (2011). Anticancer activity of thymoquinone in breast cancer cells: possible involvement of PPAR- $\gamma$ pathway. Biochem Pharmaco, 82(5): 464-475.

Zhang, Q., Bai, Q., Yuan, Y., Liu, P. and Qiao, J. (2010). Assessment of seminal estradiol and testosterone levels as predictors of human spermatogenesis. Journal of Andrology, 31 (2): 215-220. 